\title{
Meta
}

Journal des traducteurs

Translators' Journal

\section{Tennent, M. (eds) (2005) : Training for the New Millennium, Amsterdam/Philadelphia, John Benjamins, 274 p.}

\section{Álvaro Echeverri}

Volume 53, numéro 3, septembre 2008

URI : https://id.erudit.org/iderudit/019250ar

DOI : https://doi.org/10.7202/019250ar

Aller au sommaire du numéro

Éditeur(s)

Les Presses de l'Université de Montréal

ISSN

0026-0452 (imprimé)

1492-1421 (numérique)

Découvrir la revue

Citer ce compte rendu

Echeverri, Á. (2008). Compte rendu de [Tennent, M. (eds) (2005) : Training for the New Millennium, Amsterdam/Philadelphia, John Benjamins, 274 p.] Meta, 53(3), 691-697. https://doi.org/10.7202/019250ar

Ce document est protégé par la loi sur le droit d'auteur. L'utilisation des services d'Érudit (y compris la reproduction) est assujettie à sa politique d'utilisation que vous pouvez consulter en ligne.

https://apropos.erudit.org/fr/usagers/politique-dutilisation/
Cet article est diffusé et préservé par Érudit.

Érudit est un consortium interuniversitaire sans but lucratif composé de l'Université de Montréal, l'Université Laval et l'Université du Québec à Montréal. Il a pour mission la promotion et la valorisation de la recherche. https://www.erudit.org/fr/ 
Tennent, M. (eds) (2005): Training for the New Millennium, Amsterdam/Philadelphia, John Benjamins, 274 p.

La nature interdisciplinaire de la traductologie permet que le phénomène de la traduction soit analysé sous le prisme de diverses disciplines. Les universitaires évoluant dans des disciplines telles que les études littéraires, la philosophie, l'enseignement des langues, la linguistique, la sociologie, les études bibliques et même les sciences naturelles se rencontrent dans les mêmes espaces de discussion avec des professionnels de la traduction ou de l'interprétation. Cette diversité de parcours, d'intérêts scientifiques et d'expériences professionnelles trouve dans le contexte universitaire deux points de convergence principaux. D'abord, tous ces professionnels et universitaires essaient de mieux comprendre et de mieux décrire le même phénomène: la traduction. Ensuite, et à différents degrés d'engagement, bon nombre d'entre eux sont les responsables de la formation des futurs langagiers.

Le développement et la diversification des professions langagières donnent lieu à de nouveaux défis qui demandent de ces formateurs des connaissances linguistiques et disciplinaires, une expérience professionnelle et certainement un savoir pédagogique. Si l'on en croit l'ouvrage collectif dirigé par Martha Tennent, l'enseignement efficace de la traduction devra passer par une révision des méthodologies traditionnelles centrées sur l'enseignant qui, selon elle (p. xxiv), ne s'avèrent plus adéquates car elles n'engagent pas suffisamment les apprenants dans le processus d'apprentissage. Derrière cette inadéquation se cacherait une absence chronique d'innovation dans l'enseignement de la traduction. En effet, bon nombre d'enseignants continuent à enseigner la traduction de la même manière que leurs collègues le faisaient il y a soixante ans. Il se peut que tout simplement ils ne connaissent pas d'autres façons de faire.

Les douze articles de ce collectif sont le produit des discussions tenues au Forum sur la pédagogie de la traduction réalisé en 1999 à l'École de Traduction et d'Interprétation de l'Université de Vic en Espagne. Sauf dans le cas de Rosemary Arrojo, les textes publiés ont été écrits par des traductologues enseignant dans des universités européennes. La publication inclut une préface écrite par Eugene A. Nida, une introduction de la directrice, Martha Tennent, et douze chapitres organisés en quatre parties. Le livre s'adresse aux traducteurs et aux enseignants de traduction, et couvre des sujets tels que la description du matériel, les approches pédagogiques, l'organisation des cours, la TAO, la place de la théorie dans la formation, entre autres.

La première partie du livre: Training programmes: The current situation and future prospects, comprend deux chapitres écrits par Margherita Ulrych et Helge Niska. Dans le premier, "Training translators: programmes, curricula, practices», Ulrych présente les résultats d'une enquête menée auprès des institutions d'éducation supérieure offrant une formation en traduction ou en interprétation. Le but de cette enquête était de faire le point sur le développement des pratiques de formation de traducteurs et d'interprètes ainsi que sur le degré d'adéquation de ces pratiques aux défis que les futurs langagiers doivent relever dans un monde en constante et rapide évolution. Un questionnaire, dont les lecteurs pourront trouver une copie à la fin du chapitre (p. 24) a été envoyé à 65 institutions en Europe et en Amérique du Nord. Grâce à la collaboration de 41 institutions qui ont répondu à cette enquête, l'auteure a pu confirmer un décalage entre les pratiques pédagogiques actuelles et les exigences réelles du marché de la traduction. Dans ce sens, l'auteure conclut que les traductologues ont un long chemin à parcourir avant de pouvoir affirmer que la formation des langagiers dans les institutions d'éducation supérieure correspond aux pratiques professionnelles actuelles. De la même manière, l'auteure a pu constater que les institutions chargées de la formation des langagiers sont encore loin d'accepter que l'enseignement de la traduction constitue un défi pédagogique important. 
Dans le deuxième chapitre "Training interpreters: programmes, curricula, practices", Helge Niska fournit un aperçu des principaux programmes de formation d'interprètes dans le monde. Ce travail constitue ainsi une excellente source d'information pour les personnes intéressées à suivre une formation universitaire en interprétation de conférence et pour les nouveaux venus en traductologie intéressés à la formation des interprètes. En plus de sa propre classification des modèles ou approches pour la formation des interprètes (le modèle continental, le modèle britannique/libéral, le modèle centré sur le marché et le modèle scandinave/souple), Niska présente un annuaire des programmes de formation dans quelques pays européens et un tableau de classification des programmes de formation selon le système d'évaluation mis en place par l'AIIC (Association internationale des interprètes de conférence). Dans ce deuxième chapitre, les renseignements de nature factuelle l'emportent sur les discussions pédagogiques et théoriques. Nonobstant, l'auteure analyse deux aspects de l'interprétation qui montrent la voie des développements à venir. Le premier est l'importance croissante des nouvelles technologies dans le travail des interprètes et le deuxième la consolidation et l'institutionnalisation de l'interprétation en milieu communautaire comme réponse à une demande accrue de communication interlinguale. L'étude de Niska permet aussi de constater que l'utilisation de la traduction à vue et des exercices d'interprétation reste une pratique courante dans les épreuves d'admission aux programmes d'interprétation. Ce qui veut dire que pour devenir interprète, il faut démontrer qu'on en est déjà un!

La deuxième partie du livre, Pedagogical strategies, est composée de cinq chapitres. Dans son article, «Minding the process, improving the product: Alternatives to traditional translator training», María González Davies s'intéresse à un aspect complètement absent de la littérature sur l'enseignement de la traduction: la dynamique des salles de classe. Lauteure propose une approche communicative et interactive de l'enseignement de la traduction, considérant qu'il faut explorer d'autres manières de former les traducteurs tout en partageant les initiatives et en multipliant les recherches expérimentales. L'auteure est convaincue que même s'il n'existe pas une méthode idéale pour la formation des langagiers, certaines approches permettent aux étudiants de s'engager plus activement dans leur formation. Ces approches leur permettraient de mieux comprendre et le processus d'apprentissage et le processus de traduction. La mise en place de ces approches demande deux grands types d'activités: les activités pédagogiques et les activités professionnelles. Les premières visent le développement des aptitudes nécessaires à la réalisation d'une performance traductionnelle complexe et les secondes la préparation des langagiers aux situations susceptibles de se présenter dans leur vie professionnelle. Puisant dans des concepts bien connus des sciences de l'éducation tels que les styles d'apprentissage et les styles d'enseignement, González Davies propose des cours centrés sur l'étudiant pour favoriser l'interaction et encourager l'autonomie des apprenants. Le travail de González Davies s'appuie sur les résultats des recherches menées dans le domaine de l'enseignement des langues étrangères et souligne qu'une formation pédagogique des instructeurs ne peut être que bénéfique pour la formation des traducteurs.

Francesca Bartrina et Eva Espasa offrent un chapitre, "Audiovisual translation », dans lequel elles proposent un modèle de cours de traduction audiovisuelle organisé de manière progressive en trois grands blocs: la traduction d'ouvrages dramatiques, le doublage et le sous-titrage. Les auteures se servent du concept de constrained translation (traduction conditionnée) pour parler des traductions dont le texte écrit n'est qu'une des composantes du message ou ne constitue qu'une étape intermédiaire d'un discours lu à haute voix ou prononcé sur scène (p. 83). En effet, la composante linguistique est conditionnée par d'autres éléments tels que le son, l'image, l'espace, le temps, le mouvement. Les auteures discutent les caractéristiques fondamentales de ce type de traduction, proposent des manières pour en organiser un cours et offrent des idées très intéressantes sur la manière de mener des ateliers sur le doublage et le sous-titrage. Ce chapitre constitue un excellent exemple d'une 
manière forte adéquate de combiner les aspects professionnels, techniques et pédagogiques dans les cours de traduction audiovisuelle ainsi que d'encourager la collaboration entre les enseignants.

Le cinquième chapitre de cette deuxième partie, "Computer-assisted translation", revient à Richard Samson. D’après Samson, pour les enseignants de traduction, la TAO se réduit fondamentalement aux mémoires de traduction et à quelques systèmes de bases de données qui peuvent être consultées en ligne. Selon lui, il faudrait élargir le concept de TAO à d'autres applications plus générales sans lesquelles il serait impossible aux langagiers de développer des aptitudes spécialisées. La TAO dans les programmes de formation des langagiers devrait inclure la configuration du poste de travail, la gestion des archives, le traitement de textes et l'utilisation d'Internet. Pour ce faire, Samson formule quatre recommandations: a) les programmes doivent être à la fois pratiques et variés; b) la composante informatique de la formation des langagiers doit s'étendre tout au long du cursus et ne pas se limiter à un seul cours isolé; c) la formation adéquate des instructeurs; et d) la création de partenariats commerciaux entre les institutions de formation et l'industrie des outils de TAO. Dans ce chapitre, Samson propose aussi une méthodologie d'enseignement, des exemples de projets à réaliser dans les cours de traductique et des exemples d'applications qui peuvent être utilisées en interprétation, en traduction littéraire et en traduction audiovisuelle. Samson invite également les institutions de formation à former leurs propres instructeurs à l'utilisation des outils informatiques. Selon lui, le fossé technologique intergénérationnel ne cesse de se creuser. Il arrive que face aux défis technologiques de notre temps, certains enseignants se sentent bien plus désorientés que leurs étudiants (p. 115).

Il est tacitement accepté qu'une personne possédant un diplôme universitaire et une excellente connaissance d'au moins deux langues puisse enseigner à traduire. D'après Daniel Gile, ce ne serait pas le cas de l'enseignement de l'interprétation car: «virtually all training programs are built on interpreting exercices, in both consecutive and simultaneous, with some sight translation and some peripheral exercises». La formation des interprètes serait donc la tâche exclusive des interprètes professionnels. Toutefois, le manque d'interprètes professionnels désireux de travailler dans l'enseignement fait que la formation est assurée par des non-interprètes qui ne connaissent pas le marché de l'interprétation ni ne sont en mesure de transmettre aux apprenants les stratégies ou les comportements qui déterminent la pratique professionnelle optimale. Dans son chapitre, «Teaching conference interpreting: A contribution », Daniel Gile fait le point sur quelques considérations didactiques et sur les différences qu'il faut établir entre la formation d'interprètes et la formation de traducteurs. Il explique que cet écart s'établit en termes de maîtrise des langues passives/actives et compétences cognitives. Par rapport au contenu des programmes de formation, Gile propose une progression organisée selon les différents types d'interprétation qui commence par l'interprétation consécutive sans notes et finit avec la traduction à vue. Pour ce qui est de l'évaluation, Gile propose un système de "checkpoints» qui se résume à un examen d'admission, une épreuve de mi-parcours et un examen de fin d'études. Gile parle des difficultés d'adaptation des méthodes et des principes dans des environnements dits «non standard». Un environnement non standard, selon Gile, serait celui dans lequel: a) la mâ̂trise des langues de travail chez les instructeurs n'est pas idéale; b) le nombre d'interprètes professionnels travaillant comme instructeurs est insuffisant; et c) il existe une pénurie d'équipements. Gile se prononce aussi sur la place de la théorie dans la formation des interprètes en affirmant que le contenu théorique devrait se réduire aux fondements philosophiques de l'interprétation et à sa dimension cognitive dans laquelle il faut inclure la maîtrise des langues. L'auteur affirme en toute humilité (p. 149) que tout cela a déjà été dit dans ses publications antérieures, trop nombreuses pour les inclure dans la bibliographie. Une des conclusions auxquelles arrive l'auteur est bien inquiétante: "High proficiency in graduates may result, to a large extent, from strict standards at admission and/or graduation, not from 
good training methods» (p. 149). Autant dire que les cours d'interprétation n'auraient que très peu d'incidence sur la performance des interprètes et que les candidats n'ont que très peu de possibilités d'améliorer leurs performances grâce à leurs études! Les formateurs d'interprètes dans le monde ont des raisons de s'inquiéter.

Mais si, selon Gile, du côté de l'interprétation de conférence, la formation ne paraît pas avoir un grand impact sur la performance des interprètes, du côté de l'interprétation en milieu communautaire, il semble qu’on bénéficie de vents plus favorables. Dans son chapitre, "Training interpreters to work in the public services", Ann Cornelis offre un excellent portrait de la situation de l'interprétation en milieu communautaire au Royaume-Uni et ailleurs dans l'Union européenne. La définition et l'application de normes d'excellence en interprétation communautaire deviennent indispensables si l'on tient compte que les interprètes communautaires doivent fonctionner dans des milieux tels que la justice, la santé, l'immigration et l'industrie où les décisions peuvent avoir des conséquences significatives sur le sort des personnes et des institutions. Cornelis passe en revue les critères de sélection des candidats, propose une dynamique constructive de l'apprentissage, offre une synthèse des propositions de contenus des cours et présente des exercices à réaliser dans les salles de classe. Elle insiste également sur l'importance de la connaissance et de l'application d'un code déontologique et sur le besoin d'une formation continue. En plus de constituer une excellente référence pour ceux qui s'intéressent à la formation d'interprètes en milieu communautaire, le chapitre de Cornelis permet de constater le développement vertigineux d'une activité qui, il y a quelques années, était considérée comme «le parent pauvre» de l'interprétation.

La troisième partie de cet ouvrage collectif porte sur l'importance de la théorie dans l'enseignement. Dans le chapitre "Theory and translation training", Francesca Bartrina propose le modèle d'un cours de théorie de la traduction dans lequel elle expose les objectifs et les contenus, quelques considérations méthodologiques par rapport aux cours en ligne et le plan. La pertinence de la théorie de la traduction dans la formation universitaire des langagiers ne devrait plus faire l'objet de discussions disciplinaires; elle est indiscutable. Les progrès de la traductologie devraient nous amener plutôt à nous demander non si la théorie est utile aux traducteurs mais quelles théories sont pertinentes pour quels types de traducteurs? Les traducteurs littéraires, par exemple, aborderont des théories, de prime abord pas très pertinentes pour les traducteurs de textes techniques ou scientifiques. Les détracteurs de la théorie du sens, par exemple, ne peuvent pas nier la pertinence de celle-ci dans la formation des interprètes; elle a fait ses preuves. De même, et compte tenu des courants traductologiques contemporains, on se pose davantage des questions sur des postulats théoriques affirmant que dans la traduction de textes littéraires il faudrait traduire le sens. C'est ce que Delisle semble avoir très bien compris il y a plus de vingt ans.

Dans "Causality in translator training», Andrew Chesterman aborde la traductologie sous l'angle de la causalité. Chesterman distingue trois modèles dans la théorie contemporaine de la traduction: un modèle statique, la traduction est un produit décrit par la relation entre deux textes: l'équivalence. Un modèle dynamique, la traduction considérée comme un processus qui se développe dans le temps et qui se définit par la succession des étapes d'analyse, de transfert et de restructuration. Le dernier serait un modèle causatif qui traite les traductions et les traducteurs non seulement comme les produits d'une cause ou d'une action, mais aussi comme des agents de changement et des créateurs d'effets. Après avoir introduit le concept de causalité et sa présence en traductologie, Chesterman analyse les faiblesses méthodologiques de la recherche et de la formation des traducteurs. La première de ces faiblesses est l’absence de liens entre les différents modèles théoriques. La deuxième est un manque de concision que Chesterman associe à l'absence d'hypothèses (interprétatives, descriptives, explicatives, prédictives) susceptibles d'être validées et reproduites. La troisième faiblesse identifiée par Chesterman est le manque d'intérêt à tester adéquatement les quelques hypothèses explicites existantes. L'auteur analyse ensuite si ces mêmes faiblesses 
se trouvent dans la formation des langagiers et il le fait en termes de cohérence (relation entre la théorie et la pratique, relation entre les différents cours enseignés, entre les conseils offerts par les enseignants, etc.), de concision (est-ce que les théories, les normes, les stratégies trouvent une réalisation concrète dans la pratique?); de validation des hypothèses (les apprenants ont-ils la possibilité de tester des hypothèses prescriptives? est-ce que les corrections des enseignants sont valides et représentatives?). L'auteur affirme également que l'application d'un modèle causatif de la traduction aux besoins des usagers (élèves, enseignants, traducteurs) met en évidence la portée sociale de la recherche traductologique, un cadre de recherche dans lequel les différentes hypothèses peuvent être formulées et testées. D’après Chesterman, il ne s'agit pas d'adopter les méthodes de recherche typiques des sciences naturelles et de laisser de côté les racines philosophiques, herméneutiques et littéraires qui caractérisent la traductologie. Il s'agit plutôt de passer plus de temps à démontrer les applications pratiques du riche bagage conceptuel dont jouit la traductologie. En procédant de cette manière, on acquerra une meilleure compréhension de la traduction et, partant, on pourra fonctionner sur des bases plus solides dans la formation des traducteurs.

C’est précisément par une discussion sur la compétence pédagogique des instructeurs de traduction que Christiane Nord ouvre le troisième chapitre de la troisième partie, "Training functional translators", de cet ouvrage collectif. En effet, le développement de la traduction est tel qu'aujourd'hui un diplôme universitaire est une condition essentielle pour entrer sur le marché du travail. Mais qui sont responsables de la formation de ces professionnels? Selon Nord, la formation institutionnelle des instructeurs de traduction est inexistante. Les personnes qui désirent postuler à un poste de professeur universitaire dans un programme de traduction n'ont pas besoin de qualifications pédagogiques particulières. De plus, si tel était le cas, les candidats ne sauraient peut-être pas où se procurer de telles qualifications (p. 209). Nord fait aussi référence à la situation des instructeurs débutants qui, en l'absence d'une formation pédagogique, se voient obligés d'imiter leurs propres enseignants, au moins ceux qu'ils apprécient le plus. (Voilà qui, en termes de causalité, selon Chesterman, constitue une bonne hypothèse explicative: le manque d'innovation dans l'enseignement de la traduction est un effet direct du caractère déficitaire de la formation pédagogique spécifique chez les instructeurs.) En plus de cette mise en garde de Nord, le chapitre est, en quelque sorte, l'héritage qu'elle veut léguer à la traductologie et plus précisément à l'enseignement de la traduction. Comme l'auteure même l'exprime dans son texte, elle ne souhaite pas voir enterrées avec elle toutes les connaissances amassées au cours de 35 ans d'expérience (p. 209). Aussi aborde-t-elle la sélection des matériels didactiques, les méthodes d'enseignement/apprentissage, l'évaluation, l'application des jeux de rôles dans la salle de classe et ce qu'elle appelle la méthode «queue de cochon [pigtail method]» pour combler le fossé entre théorie et pratique de la traduction. En ce qui concerne la caractérisation d'un traducteur fonctionnel, l'auteure propose une liste de compétences sur lesquelles se sont mis d'accord les participants à une conférence tenue à Leipzig en 1999. Finalement, Nord insiste sur l'importance de la recherche appliquée à l'enseignement de la traduction, car pour enseigner la traduction, il ne suffit pas d'avoir une excellente connaissance des langues, d'être un linguiste reconnu ou un expert consommé en théorie de la traduction.

Dans le dernier chapitre de la troisième partie l'ouvrage collectif, «The ethics of translation in contemporary approaches to translator training", Rosemary Arrojo s'interroge sur le modèle professionnel et éthique auquel doivent aspirer les traducteurs en formation. Arrojo analyse trois ouvrages des années quatre-vingts dix qui ont proposé une base méthodologique pour la formation des traducteurs inspirée des approches linguistiques contemporaines. Arrojo analyse les travaux de Mona Baker (In Other Words, 1992), Basil Hatim et Ian Mason (The translator as communicator, 1997) et Paul Kussmaul (Training the translator, 1995). Elle arrive à la conclusion que même si les travaux de ces auteurs donnent l'impression de tenir compte des rapports entre le langage, la culture et l'idéologie, ils sont 
porteurs des conceptions essentialistes de la traduction et de son enseignement. Par essentialiste Arrojo veut dire: une vision du monde selon laquelle la signification est quelque chose de stable et est indépendante de l'histoire, de l'idéologie et de la psychologie de ceux qui sont chargés de l'interpréter. À cette vision essentialiste de la traduction, Arrojo oppose une vision non essentialiste selon laquelle le texte qu'on appelle l'original ne commence à exister qu'au moment où il est lu ou interprété par quelqu'un dans des circonstances particulières, avec des restrictions et des objectifs particuliers. Selon une vision postmoderniste du langage, la signification est inévitablement le produit d'un individu et c'est le contexte social qui lui confère sa légitimité. À la lumière d'un tel constat, une traduction ne peut être fidèle qu'à la lecture que le traducteur fait du texte source; jamais à un original fermé comme un conteneur qui garde éternellement les significations et les intentions de l'auteur. On ne peut que partager l'opinion de l'auteure dans le cas des textes riches en contenus idiologiques et idéologiques. Néanmoins, Arrojo parle d'une prétendue résistance des traducteurs professionnels à accepter le caractère universitaire de la traduction (p. 227), soi-disant parce qu'ils ne voient aucun lien entre leur pratique et certains postulats théoriques associés à la recherche universitaire. On pourra cependant répondre à Arrojo que, de nos jours, de moins en moins de praticiens nient la place de la traduction à l'université et ils comprennent mieux aujourd'hui que les objectifs des associations professionnelles ne pourront être atteints que grâce à un vrai partenariat avec les universités. Il est pourtant vrai que la vision non essentialiste de la traduction n'est pas facile à appliquer pour quelqu'un qui gagne sa vie en traduisant des "manuels de l'usager». Encore une fois, la question à se poser est: quelles théories pour quels types de textes et quelles théories pour quelles traductions?

Le texte de Michael Cronin, «Deschooling translation: Beginning of century reflections on teaching translation and interpreting» est à la fois l'épilogue et la quatrième partie de cet ouvrage collectif dirigé par Martha Tennent. Cronin prend comme point de départ la situation de l'enseignement de la traduction avant les années quatre-vingts dix. Selon lui, ni les théoriciens ni les praticiens de la traduction n'étaient en mesure d'appuyer leurs choix pédagogiques sur des théories solides de l'enseignement. Cronin met en exergue l'influence que des travaux comme ceux de Kiraly (1995) et de Kussmaul (1995) ont eue pour combler ce fossé. L’auteur souligne aussi le besoin impérieux, pour les didacticiens d'augmenter les recherches empiriques dont les résultats permettraient aux enseignants de travailler sur des bases plus solides. Cronin appelle déscolarisation (deschooling) la position des traductologues comme Tennent, Kiraly, Kussmaul, Robinson, González Davies, et d'autres, qui insistent sur la pertinence de donner l'initiative de l'apprentissage aux élèves et d'abandonner les approches pédagogiques centrées sur l'enseignant. Comme l'affirme Cronin, les approches pédagogiques sont aussi porteuses d'idéologies et leur mise en place ne doit pas se faire sans une idée exacte de leur portée et de leur pertinence; c'est la géopolitique de la pédagogie. Cronin se réfère indirectement au manque d'innovation en enseignement de la traduction lorsqu'il parle du dédain chez les enseignants pour toute prise en compte de l'évolution diachronique des étudiants. Cronin termine son chapitre en nous rappelant que les traducteurs sont, à la fois, agents et victimes des changements et qu'ils auront davantage besoin de théories appropriées pour consolider la traduction en tant que profession et discipline universitaire.

En conclusion et tenant compte des sujets abordés dans ces douze chapitres, on peut dire que les enjeux de la formation des langagiers du xxI siècle sont: la formation pédagogique des instructeurs; la multiplication des études empiriques; la collaboration entre praticiens et théoriciens et entre formateurs et employeurs. Deux considérations se retrouvent dans la majorité des chapitres de cet excellent ouvrage: le premier est le fossé pédagogique entre l'enseignement de la traduction et sa pratique, et le deuxième est le manque d'études empiriques, voire de données empiriques, pour soutenir les hypothèses interprétatives des traductologues. Comme le constate Christiane Nord (p. 209), l'expérience fait 
d'excellents enseignants. Le problème de l'expérience est son caractère personnalisé qui fait que, contrairement au savoir, elle est rarement transmissible. Le savoir pédagogique s'obtient par l'étude et par la recherche, et se conserve et s'apprend dans les livres. L'expérience pédagogique s'acquiert dans les salles de classe et, très fréquemment, elle est «enterrée avec les os».

Álvaro Echeverri

Université de Montréal, Montréal, Canada

Delabastita, D., D’hulst, L. and R. Meylaerts (eds.) (2006): Functional Approaches to Culture and Translation, Selected papers by José Lambert, Benjamins Translation Library, vol. 69, John Benjamins Publishing Company, Amsterdam / Philadelphia, xxviii $+225 \mathrm{p}$.

Le présent ouvrage, le soixante-neuvième de la série consacrée par la Benjamins Translation Library à la recherche et à la formation en traduction et interprétation, regroupe des textes rédigés de 1977 à 2004 par le professeur José Lambert. Né en 1941 dans le village de Wingene en Belgique, pays aux confins, mais aussi participant à trois cultures littéraires (française, néerlandaise et allemande), José Lambert passera une bonne partie de sa carrière à étudier les influences qu'elles ont exercées et exercent encore l'une sur l'autre par le truchement de la traduction. Tout en pouvant se réclamer de chacune de ces trois cultures, il savait aussi qu'il ne pouvait chercher son identité propre dans aucune d'elles, ce qui l'amènera à remettre en question le concept même de «littérature nationale».

Après avoir étudié la philologie romane à la Katholieke Universiteit Leuven, il obtint en 1972 un doctorat en littérature comparée, domaine qu'il continuera à enseigner à la même université. Le point tournant de sa carrière fut sans doute le colloque qu'il organisa en 1976 à Louvain sur le thème "Littérature et traduction » où, en compagnie de collègues comme André Lefevere, Raymond Van den Broeck, James S. Holmes, Susan Bassnett, Itamar Even-Zohar, Gideon Toury et Hendrik Van Gorp, il affirma que la traduction était pour le moins autant question de culture que de langage et qu'on ne pouvait l'étudier du seul point de vue de la conformité entre les textes de départ et d'arrivée.

Poursuivant sur ce thème, il lança dans les années 1980 un projet de recherche sur les relations entre traduction et littérature dans la France du XIX ${ }^{e}$ siècle, projet qu'il étendit, dans un premier temps à l'influence de la France sur l'émergence de la littérature belge et, dans un deuxième temps, à l'influence de la littérature française sur celles de pays récemment décolonisés. En 1989, il fonda avec Gideon Toury la revue Target qui devait avoir une énorme influence sur le développement des études reliées à la traduction. La même année, il mit sur pied la chaire CERA pour la traduction, les communications et la culture, laquelle prendra plus tard le nom de CETRA.

Les treize textes, regroupés dans ce volume selon leur ordre chronologique, constituent un compendium de l'évolution conceptuelle et méthodologique de celui à qui revient l'honneur d'avoir fait entrer la traduction littéraire dans le domaine de la littérature comparée.

\section{7: Traduction et technique romanesque}

Lambert s'élève ici contre le concept selon lequel une traduction se fait en fonction de petites unités comme la phrase, plutôt qu'en fonction de l'ensemble d'un texte; s'il en était ainsi, la traduction de Bacon, par exemple, ne poserait guère de problèmes différents de celle de Shakespeare. Les décisions prises par un traducteur dépendent de la nature du message à transmettre et, par conséquent, de l'interprétation globale de l'œuvre d'un auteur. Plutôt que de comparer les textes de départ et d'arrivée sous leur seul aspect linguistique, il propose 